\title{
Insulin-Like Growth Factor (IGF) Signaling through Type 1 IGF Receptor Plays an Important Role in Remyelination
}

\author{
Jeffrey L. Mason, ${ }^{1}$ Shouhong Xuan, ${ }^{2}$ Ioannis Dragatsis, ${ }^{2}$ Argiris Efstratiadis, ${ }^{2}$ and James E. Goldman ${ }^{1}$ \\ ${ }^{1}$ Department of Pathology and The Center for Neurobiology and Behavior, and ${ }^{2}$ Department of Genetics and Development, Columbia University, New York, \\ New York 10032
}

We examined the role of IGF signaling in the remyelination process by disrupting the gene encoding the type 1 IGF receptor (IGF1R) specifically in the mouse brain by Cre-mediated recombination and then exposing these mutants and normal siblings to cuprizone. This neurotoxicant induces a demyelinating lesion in the corpus callosum that is reversible on termination of the insult. Acute demyelination and oligodendrocyte depletion were the same in mutants and controls, but the mutants did not remyelinate adequately. We observed that oligodendrocyte progenitors did not accumulate, proliferate, or survive within the mutant mice, compared with wild type, indicating that signaling through the IGF1R plays a critical role in remyelination via effects on oligodendrocyte progenitors.

Key words: demyelination; oligodendrocyte; progenitors; apoptosis; macrophages; transgenic

\section{Introduction}

After exposure to chemical, mechanical, or autoimmune insults resulting in demyelination of axons within the adult CNS, oligodendrocyte progenitors have some capacity to initiate a restorative remyelination process (Ludwin, 1978; Yao et al., 1995; Gensert and Goldman, 1997). Several of the signaling molecules that influence the migration, proliferation, and differentiation of oligodendrocyte progenitors are expressed within remyelinating lesions in the CNS (Redwine and Armstrong, 1998; Hinks and Franklin, 1999; Mason et al., 2000a). However, the factor(s) responsible for the recruitment and differentiation of these progenitors in vivo is not known.

An important signaling system that has been implicated in the process of myelination consists of IGF-1 activating the type 1 receptor (IGF1R) (Liu et al., 1993). IGF-1 is a survival factor for oligodendrocytes (Barres et al., 1993; Ye and D'Ercole, 1999; Mason et al., 2000b) and a differentiation factor for neonatal (McMorris and Dubois-Dalq, 1988; Mozell and McMorris, 1991) and adult (Mason and Goldman, 2002) oligodendrocyte progenitors in vitro. Its levels are elevated within demyelinating and remyelinating lesions in the adult CNS (Liu et al., 1994; Yao et al., 1995; Mason et al., 2000a). In addition, the inability of the adult CNS to remyelinate after a demyelinating lesion in interleukin$1 \beta-/-$ mice coincides with a significant reduction in IGF-1 expression (Mason et al., 2001a). Thus, although the function of IGF-1 within demyelinating and remyelinating lesions is not clear, it seems that it may be important for inducing oligoden-

Received April 10, 2003; revised June 11, 2003; accepted July 7, 2003.

This work was supported by Grant FG 13377-A-1 to J.L.M. from the National Multiple Sclerosis Society and by National Institutes of Health Grants NS17125 to J.E.G. and CA75553 (Project 3) to A.E. We thank Bernetta Abramson for technical help and Dr. Bill Stallcup for the NG2 antibodies

Correspondence should be addressed to Dr. Jeffrey Mason, Department of Pathology, Columbia University, 630 West 168th Street, P\&S 15-420, New York, NY 10032. E-mail: jm1306@columbia.edu.

I. Dragatsis' present address: Department of Physiology, University of Tennessee, Memphis, TN 38163. Copyright $\odot 2003$ Society for Neuroscience $\quad$ 0270-6474/03/237710-09\$15.00/0 drocyte progenitors to differentiate and remyelinate the demyelinated axons.

To address the role of IGF signaling in the remyelination process directly, we used conditional mouse mutants, in which the expression of the Igflr gene was ablated by Cre-mediated recombination specifically in the CNS. We assessed, in comparison with normal siblings, the consequences of exposure of these animals to the neurotoxicant cuprizone (Matsushima and Morell, 2001), which induces a reversible demyelinating lesion in the corpus callosum of adult mice. We observed that remyelination does not adequately occur in the absence of IGF signaling and that oligodendrocyte progenitors do not proliferate or survive as well without IGF1R.

\section{Materials and Methods}

Mouse crosses. CamKII $\alpha$-cre transgenic mice (line Rlag\#5) (Dragatsis and Zeitlin, 2000) were mated with $I g f 1 r^{+/-}$heterozygotes (Liu et al., 1993) to derive among other progeny animals with a CamKII $\alpha$-cre; Igflr ${ }^{+/-}$genotype. These offspring were then crossed with homozygous conditional mutants carrying modified Igf1r alleles with loxP sites flanking exon 3 (Igfl $\left.r^{f l o x / f l o x}\right)$ (Dietrich et al., 2000) to generate the desired experimental animals (CamKII $\alpha$-cre; Igfl $\mathrm{r}^{\text {flox/- }}$ ), the genotype of which was converted by Cre-mediated recombination to IgfI $r^{\text {flox/- }}$ specifically in the brain. The CamKII $\alpha$-cre transgenic animals were also crossed with R26R reporter mice carrying a LacZ transgene in the ROSA26 locus (Soriano, 1999) that can be activated by Cre-mediated recombination.

Detection of CaMKII $\alpha$-cre recombination. We used a CaMKII $\alpha$-cre transgenic mouse and assessed cre expression using a $\beta$-gal reporter sensitive to Cre-mediated recombination in the brain (Soriano, 1999). We perfused postnatal day 1 (P1) transgenic mice intracardially with paraformaldehyde (PFA), removed the forebrains, and processed the tissue for frozen sectioning. Tissue sections were dried, rehydrated, blocked with 5\% normal goat serum (NGS), and then stained with the mouse IgM monoclonal O4 supernatant (1:5) or the rabbit polyclonal NG2 antibody (1:100; a gift from Bill Stallcup, Scripps Institute, San Diego, CA) to detect oligodendrocyte progenitors and a mouse monoclonal $\operatorname{IgG}_{1}$ antibody directed against $\beta$-gal (1:100; Promega, Madison, WI) to detect 


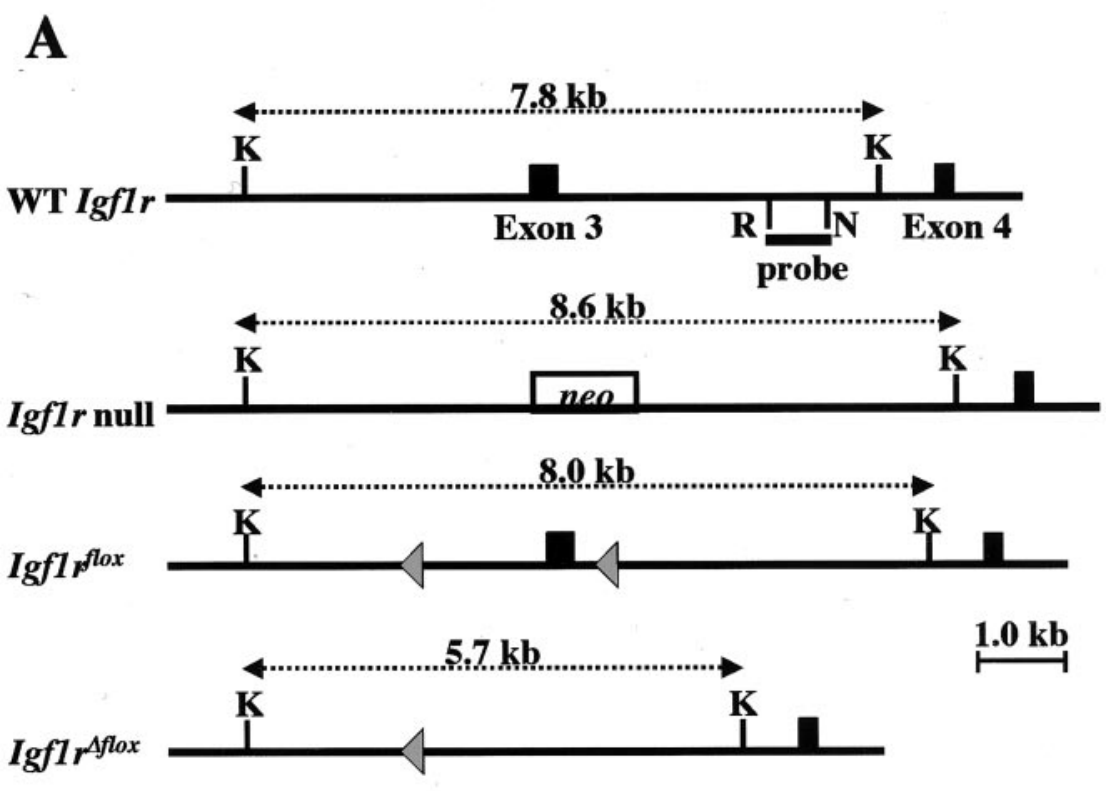

B

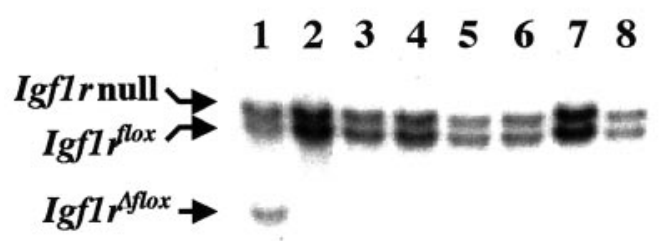

C

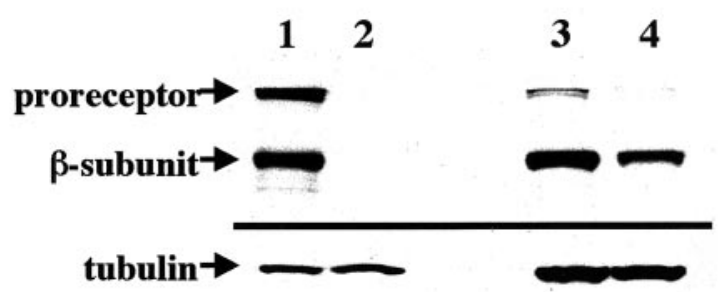

Figure 1. Partial restriction map in the region of exons 3 and 4 (black rectangles) of the mouse lgfir locus. $A$, Targeted null and also conditional mutant alleles before and after (re-mediated excision of exon 3 and flanking sequences (Igf1 flox and lgf1r $\Delta^{f l o x}$, respectively) are shown. The floxP sites (gray triangles) are not in scale. The position of a $0.8 \mathrm{~kb} E c 0 \mathrm{RV}-N \mathrm{Nil}(R-N)$ probe used for Southern analyses and the sizes of the Kpnl ( $K$ ) DNA fragments detectable by this probe are indicated. Brain-specific, (re-mediated recombination in heterozygous mice possessing floxed and null Igfir alleles and also carrying a CaMKIl $\alpha$-cre transgene is shown. WT, Wild-type. B, Example of Southern analysis of Kpnl-digested DNA that was extracted from various tissues of the same lgf1r ${ }^{f l o x}-$; CaMKII $\alpha$-cre animal: forebrain (lane 1), cerebellum (lane 2), heart (lane 3), lung (lane 4), liver (land 5), kidney (lane 6), spleen (lane 7), and testis (lane 8). Because the CaMKII $\alpha$-cre transgene is not expressed in all forebrain cells, Cre-mediated recombination and forebrain-selective appearance of a fragment characteristic for an Igf1 ${ }^{D f l o x}$ allele occurred in a fraction of all floxed loci. C, Western blot analysis of IGF1R expression. The examined protein extracts were from cultured fibroblasts prepared from wild-type (lane 1) and Igf1r null (lane 2) embryos (positive and negative control, respectively) or from mouse forebrains dissected from wild-type (lane 3) or lgflr ${ }^{\text {flox }-}$; CaMKIl $\alpha$-cre (lane 4) animals. The positions of the IGF1R precursor and $\beta$-subunit detectable by the specific antibody used (see Materials and Methods) are shown. The blot was reprobed with an anti-tubulin antiserum (loading control; bottom). Quantitation of the results (see Materials and Methods) indicated that the amount of IGF1R $\beta$-subunit in the mutant relative to wild type is $\sim 50 \%$.

Cre-mediated recombination overnight. The sections were then incubated with the appropriate rhodamine-conjugated (anti-rabbit IgG or anti-mouse IgM) and FITC-conjugated anti-mouse IgG1 secondary antibodies (1:100; Southern Biotechnology, Birmingham, AL), cover- slipped, and examined using an Olympus (Lake Success, NY) BX60 microscope equipped with epifluorescent optics.

Southern and Western analyses. The degree of Cre-mediated DNA excision was quantitated by Southern analysis, after determination of the level of hybridization signal in diagnostic fragments using a PhosphorImager (Molecular Dynamics, Sunnyvale, CA) and correcting for background hybridization.

Western blot analysis was performed from the forebrain of 4-week-old mice, as described previously (Di Cola et al., 1997), using antisera against the carboxyterminal domain of IGF1R (C20; Santa Cruz Biotechnology, Santa Cruz, CA) or against tubulin (Covance, Berkeley, CA). Immunoreactive bands were visualized with ECL detection reagents (Amersham Biosciences, Piscataway, NJ). Quantitation of luminescent signals was performed on a Kodak (Rochester, NY) Digital Science 1D 440CF Imaging Station.

Detection of IGF1R expression in adult oligodendrocyte progenitors. Cycling progenitors were isolated from the corpus callosum of adult wild-type or $\Delta I g f 1 r$ mice, as described previously (Gensert and Goldman, 2001; Mason and Goldman, 2002). Briefly, the corpus callosum was dissected from 8-week-old wild-type or $\Delta I g f 1 r$ mice, then mechanically and enzymatically dissociated with trypsin (Sigma, St. Louis, $\mathrm{MO}$ ) and collagenase (Worthington, Freehold, $\mathrm{NJ})$. The cellular suspension was filtered through sterile $0.74 \mu \mathrm{m}$ mesh, and the trypsin was neutralized with $10 \%$ heat-inactivated serum. Cells were collected by centrifugation and resuspended in $0.9 \mathrm{M}$ sucrose/MEM. This cellular suspension was centrifuged, and the cycling progenitors (bottom layer) were collected and resuspended in serum-free media (d-Biotin; 10 $\eta \mathrm{g} / \mathrm{ml}$; Sigma), insulin ( $5 \mu \mathrm{g} / \mathrm{ml}$; Collaborative Research, Bedford, MA), progesterone (20 $\eta \mathrm{M}$; Sigma), putrescine (100 $\mu \mathrm{M}$; Sigma), selenium (5 $\eta \mathrm{g} / \mathrm{ml}$; Collaborative Research), transferrin (50 $\mu \mathrm{g} / \mathrm{ml}$; Sigma), glutamine (2 mM; Invitrogen, Carlsbad, CA), HEPES buffer (15 mm; Sigma), 3,3,5-triiodo-L-thyronine (15 $\eta \mathrm{M}$; Sigma), penicillin/streptomycin (100 U/100 $\mu \mathrm{g} / \mathrm{ml}$; Invitrogen), and BSA (1 $\mathrm{mg} / \mathrm{ml}$; Sigma) in DMEM/F12 (Invitrogen).

Cells were stained for O4 and IGF1R immediately after they were plated. The cells were fixed with $4 \%$ PFA, incubated with $20 \%$ NGS for 30 min to block nonspecific binding, and then incubated with mouse monoclonal IgM O4 supernatant (1:5) and mouse monoclonal $\mathrm{IgG}_{1}$ antiIGF1R (1:200; Upstate Biotechnology, Lake Placid, NY) overnight at $4^{\circ} \mathrm{C}$. The anti-IGF1R antibody from Upstate Biotechnology was used for immunohistochemistry, because the anti-IGF1R antibody from Santa Cruz Biotechnology used for Western blot analysis does not work well for this type of analysis. The cells were then incubated with a combination of FITC-conjugated goat anti-mouse IgM and TRITC-conjugated goat anti-mouse IgG $\mathrm{G}_{1}$ antibodies (1:100; Southern Biotechnology) for $1 \mathrm{hr}$ at room temperature, counterstained with 4,6-diamidino-2-phenylindole (DAPI; Molecular Probes, Eugene, OR), coverslipped, and examined using an Olympus BX60 microscope equipped with epifluorescent optics. Cells were isolated, stained, and 
counted from the forebrain of three separate wildtype and $\Delta I g f l r$ mice. Two hundred $\mathrm{O}^{+}{ }^{+}$cells from each experiment were counted.

Induction of demyelination/remyelination. $\Delta \operatorname{Igfl} 1 \mathrm{r}$ mice were bred and maintained in our animal facility at Columbia University. At 8 weeks of age, $\Delta I g f 1 r$ and littermate wild-type male mice were fed a diet of milled Purina mouse chow containing $0.2 \%$ cuprizone (Sigma) by weight for up to 6 weeks to induce demyelination. Subsequently, mice were returned to a normal diet for another 3-6 weeks to allow for remyelination (Hiremath et al., 1998). All animal procedures were conducted in accordance with guidelines approved by the Institutional Animal Care and Use Committee and the Columbia Division of Laboratory Animal Medicine.

Immunohistochemistry. We anesthetized and intracardially perfused mice with PFA, removed the brains, and processed the tissue for frozen sectioning, as described previously (Mason et al., 2000a). All comparative analyses were focused at the fornix region of the corpus callosum corresponding to sections $220-260$ of the mouse brain atlas (Sidman et al., 1971).

Tissue sections were dried, rehydrated, blocked with 5\% NGS, and then incubated overnight with the primary antibody(s) diluted in the blocking solution. The sections were then incubated with the appropriate FITCconjugated secondary antibody (1:100; Southern Biotechnology), counterstained with DAPI (Molecular Probes), coverslipped, and examined using an Olympus BX60 microscope equipped with epifluorescent optics.

Antibodies. A mouse monoclonal $\operatorname{IgG}_{2 \mathrm{~B}}$ antibody directed against MBP (1:1000; Sternberger Monoclonals) was used to detect myelin. A mouse monoclonal $\operatorname{IgG}_{1}$ antibody directed against phosphorylated and nonphosphory-

lated high molecular weight neurofilaments (NF-H; 1:1000; Sternberger Monoclonals) was used to detect axons. The mouse monoclonal $\operatorname{IgG}_{2 \mathrm{~B}}$ antibody CC1 (1:10; Oncogene, Uniondale, NY) was used as a marker for mature oligodendrocytes (Messersmith et al., 2000). The rabbit polyclonal NG2 antibody (1:100) was used as a marker for oligodendrocyte progenitors (Nishiyama et al., 1996). The mouse monoclonal $\operatorname{IgG}_{2 \mathrm{~A}}$ antibody F4/80 (1:10; Serotec, Indianapolis, IN) was used to detect microglia/macrophages. The rabbit polyclonal KI-67 antibody (1:1000; Santa Cruz Biotechnology) was used to detect proliferating cells. The goat polyclonal anti-tumor necrosis factor- $\alpha$ (TNF- $\alpha$ ) antibody (1:100; R \& D Systems, Minneapolis, MN) was used to detect TNF- $\alpha$.

Terminal deoxynucleotidyl transferase-mediated biotinylated UTP nick end labeling (TUNEL) assay. The TUNEL assay was used to detect cells undergoing apoptosis using the NeuroTACS II assay kit (Trevigen, Gaithersburg, $\mathrm{MD})$. Frozen tissue sections were incubated with either the NG2 or CC1 antibody as described above. The tissue sections were then incubated with $1 \times$ terminal deoxynucleotidyltransferase labeling buffer for $5 \mathrm{~min}$ and then processed for biotin-dNTP-labeling of fragmented DNA, according to the manufacturer's instructions. The tissue sections were then incubated with a fluorescein-conjugated rat anti-mouse $\operatorname{IgG}_{2 \mathrm{~b}}$ diluted in a $2 \%$ streptavidinTexas Red complex (Vector Laboratories, Burlingame, CA)/PBS, counterstained with DAPI, coverslipped, and examined using an Olympus BX60 microscope equipped with epifluorescent optics.

Cell number quantification. Immunopositive cells were obtained by counting only those cells with an identified nucleus, observable by DAPI staining, within the medial region of the corpus callosum. Individual cell counts were conducted on both sides of midline, each corresponding to an area of $0.033 \mathrm{~mm}^{2}$. The cell counts from both areas were averaged to give a total for each tissue section. Cell counts from three nonadjacent tissue sections for each animal were then averaged to give a representative cell count for each animal in the study. Because of the high density of $\mathrm{NG}_{2}{ }^{+}$cells and their processes at 4 weeks of cuprizone treatment, a Leica (Nussloch, Germany) TCS-NT Laser Scanning microscope using $100 \times$ oil objective and a pinhole size of 1.0 Airy disk units was used to obtain cell counts for the $\mathrm{NG}_{2}{ }^{+}$cells. The cell counts are presented in the text as the mean and SEM from at least three to four mice at each time point.

Statistical analysis. Statistical comparisons were made using a onefactor between subjects ANOVA, followed by Tukey's test to assess significance among groups.

\section{Results}

\section{Ablation of IGF1R function in oligodendrocyte progenitors} Tissue-specific conditional mutagenesis using the cre/loxP recombination system (Sauer, 1998; Rossant and McMahon, 1999) requires crosses between Cre-producing and Cre-responding strains of mice. Responders used in this study, in which exon 3 of the Igf $1 r$ gene is "floxed" (flanked by loxP sites in direct orientation; Fig. 1), have been described previously (Dietrich et al., 2000). To drive cre expression in the oligodendrocytes of producer mice, we used CaMKII $\alpha$-cre transgenic mice (Dragatsis and Zeitlin, 2000). Southern and Western analyses (Fig. 1B,C) yielded results consistent with the information provided by the use of the LacZ reporter. Thus, Cre-mediated DNA excision in $\Delta \operatorname{Igflr}$ mice was detected in forebrain (and at very low levels in the cerebellum and the testis, in which the cre transgene is mar- 
$\mathbf{A}$
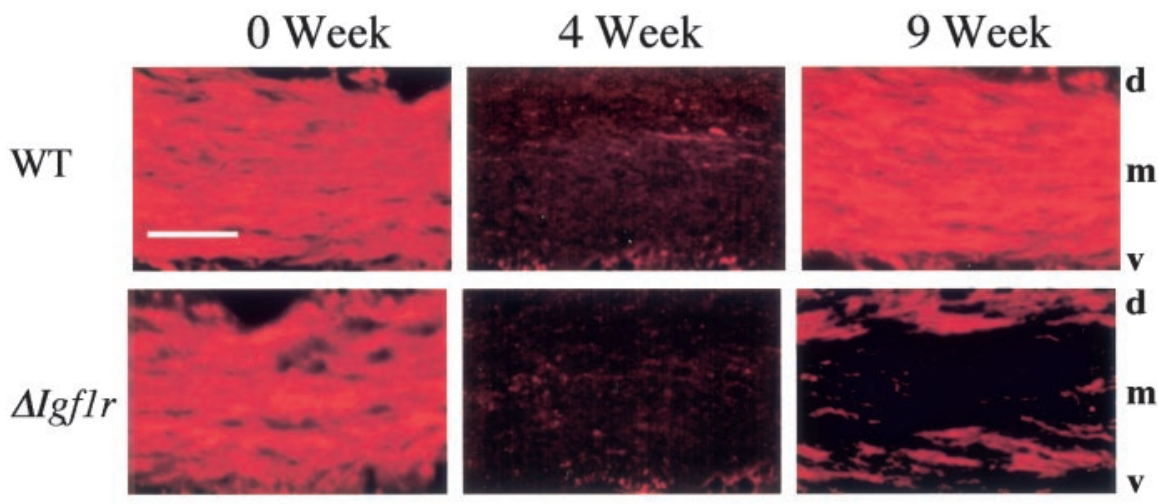

B
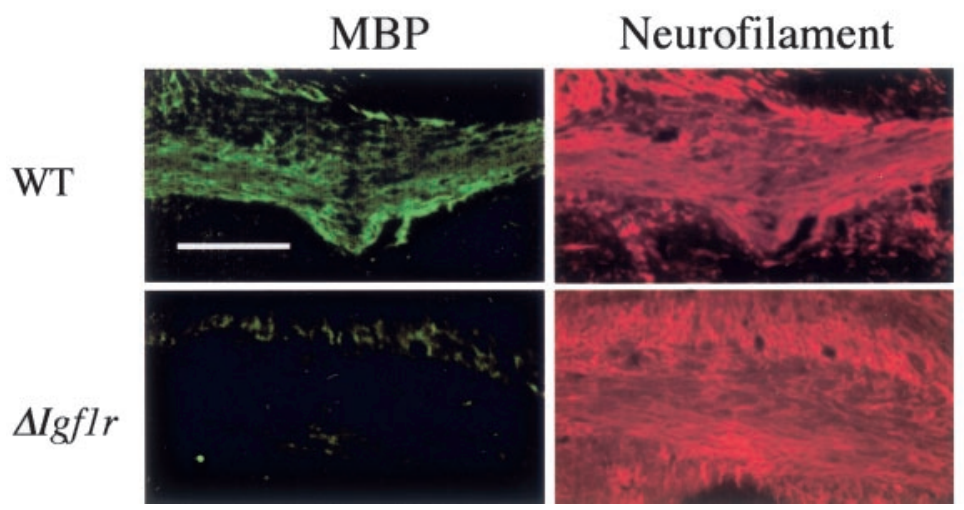

Figure 3. Effects of IGF signaling on demyelination and remyelination. $A$, To determine whether IGF signaling affects demyelination and remyelination, tissue sections from three to four wild-type (WT) and $\Delta / g f 1 r$ mice at each time point were stained for MBP (red). Representative micrographs are shown. Demyelination is complete by 4 weeks in both WT and $\Delta / g f 1 r$ mice. However, the middle ( $\mathrm{m}$ ) region of the corpus callosum fails to remyelinate by 9 weeks in $\Delta / g f 1 r$ mice, whereas the ventral (v) and dorsal (d) edges of the corpus callosum are remyelinated. $B$, To determine whether the lack of remyelination observed in the middle region of the corpus callosum in $\Delta / g f 1 r$ mice is because of lost or damaged axons, we stained sections from three to four wild-type (WT) and $\Delta / g$ fir mice for the presence of MBP (green) and neurofilaments (red) at 9 weeks. There appears to be no loss or damage of axons within this demyelinated area. Scale bars: $A, 10 \mu \mathrm{m} ; B, 50 \mu \mathrm{m}$.

ginally expressed) (Dragatsis and Zeitlin, 2000), whereas in all other examined tissues the floxed allele had remained intact (Fig. $1 B)$. The near absence of Cre-mediated recombination in the cerebellum suggests intrinsic differences among neural cells within different regions of the brain in regard to CaMKII $\alpha$ expression. Quantitation (see Materials and Methods) indicated that $\sim 45 \%$ of the Igf1 flox allele was converted to Igf1r $\Delta^{\text {flox }}$ (the cre transgene is not expressed in all forebrain cell constituents). In close agreement with the results of Southern analysis, Western blotting showed that IGF1R protein was reduced to $\sim 50 \%$ of normal in the forebrain of adult conditional mutants (Fig. 1C).

Although it has been reported that, in contrast to neurons, glial cells do not express CaMKII $\alpha$ (Vallano et al., 2000), we observed that in the particular CaMKII $\alpha$-cre transgenic line used in our analysis (R1ag5), the CaMKII $\alpha$ promoter was active and able to expresses cre, not only in $90 \%$ of neurons in the adult forebrain (Dragatsis and Zeitlin, 2000), but also in oligodendrocyte progenitors. This localization was demonstrated by generating and analyzing bitransgenic mice carrying the CaMKII $\alpha$-cre transgene and, in addition, a $\beta$-galactosidase (LacZ) transgenic reporter (Soriano, 1999) that becomes functional only after Cre- mediated recombination (activity can be monitored by X-gal staining). When forebrain sections from such bitransgenic neonates (P1) were stained to assess $\beta$-gal expression in O4-positive and NG2positive oligodendrocyte progenitors, we observed that the cells were also $\beta$-gal positive (Fig. $2 \mathrm{~A}$ ), demonstrating that Cre is, indeed, expressed and is able to exert its recombinogenic action in oligodendrocyte precursors during development. These results suggested that, on expression of the recombinase from the CaMKII $\alpha$-cre transgene, both oligodendrocyte progenitors and mature oligodendrocytes should become devoid of IGF signaling by conversion of the $I g f 1 r^{f l o x /-}$ genotype to $\operatorname{Igf1r} \Delta^{\text {flox/- }}$. For simplicity, these experimental mice will be referred to below as $\Delta \operatorname{Igfl} r$ mice, although in all cells other than those expressing Cre the floxed allele of the Igflr gene remains intact.

As a test for Cre activity in oligodendrocyte progenitors of the adult CNS, we isolated $\mathrm{O}^{+}{ }^{+}$cells from the corpus callosum of $\Delta I g f 1 r$ mice and compared the expression of IGF1R in these mice compared with $\mathrm{O}_{4}{ }^{+}$cells from wild-type mice using immunohistochemistry, as described previously (Mason and Goldman, 2002). We found that $91 \pm 3.1 \%$ of the wild-type cells expressed IGF1R, whereas only $6 \pm 2.1 \%$ of the cells from $\Delta I g f 1 r$ mice expressed the receptor (Fig. $2 B$ ). Thus, the genetic strategy successfully excises the IGF1R from oligodendrocyte progenitors in both neonates and adults.

In contrast to mice globally lacking IGF1R, which die at or soon after birth because of respiratory failure (Liu et al., 1993), the $\Delta$ Igflr mice survived to adulthood and did not exhibit any overt morphological or behavioral abnormalities. In particular, the degree of myelination appeared to be normal in adult $\Delta I g f 1 r$ mice (Fig. $3 A$ ), suggesting that IGF signaling may not play a significant role in the myelination process during development. Alternatively, any such role could have been compensated effectively by some other signaling pathway. On the basis of these results, we decided that the $\Delta I g f 1 r$ mice are suitable models that would allow us to investigate the functional significance of IGF signaling during demyelination and remyelination.

IGF signaling plays a critical role in remyelination of the adult murine CNS

Cuprizone induces a consistent and reversible demyelinating lesion within the medial region of the corpus callosum of adult mice (Mason et al., 2001b). This demyelination occurred to the same extent in both wild-type and $\Delta I g f 1 r$ mice by 4 weeks, as assessed by the absence of MBP (Fig. $3 A$ ). Subsequent remyelination of the corpus callosum in wild-type mice begins at 6 weeks and is completed by $9-10$ weeks if the mice are returned to a normal diet after 6 weeks of cuprizone (Fig. 3A) (Mason et al., 2001b). In contrast, remyelination was not observed in the middle 
region of the corpus callosum in $\Delta I g f 1 r$ mice by 9 weeks (Fig. $3 A$ ) or 14 weeks (data not shown). We noted, however, a thin zone of remyelination in the extreme ventral and dorsal edges of the callosum (see Discussion). This failure of remyelination in the middle region of the corpus callosum was a reproducible phenomenon, observed in all four of the $\Delta I g f 1 \mathrm{r}$ mice examined. The lack of remyelination at 14 weeks suggests that remyelination is not simply delayed in the $\Delta$ Igf1r mice.

To determine whether the lack of remyelination in $\Delta I g f 1 r$ mice might be because of a loss of axons within this region, we stained for MBP (myelin) and NF-H (axons) at 9 weeks. The results from three separate mice demonstrated that axons were still present within the middle region of the demyelinated corpus callosum (Fig. 3B).

\section{The accumulation of}

microglia/macrophages and TNF- $\alpha$ corresponds spatially with

demyelination and persists in the absence of IGF signaling

Microglia/macrophages clearing myelin debris by phagocytosis accumulate within the demyelinating corpus callosum in wild-type mice between 2 and 5 weeks (Hiremath et al., 1998). Also present during the same period is the cytotoxic cytokine TNF- $\alpha$, which is expressed by microglia/macrophages and astrocytes (Arnett et al., 2001). TNF- $\alpha$ facilitates the continued activation of microglia/macrophages and promotes the proliferation of oligodendrocyte progenitors to restore the lesion (Arnett et al., 2001). Thus, for further assessment of the demyelination process, we examined histologically the demyelinated region for the presence of TNF- $\alpha$ and of microglia/macrophages, the latter identified by the F4/80 antibody. We observed that the accumulation of these two indicators at 4 weeks was the same in both wild-type and $\Delta I g f 1 r$ mice (Fig. 4). In contrast, a significant difference was observed at 9 weeks. At this time, the microglia/ macrophages and TNF- $\alpha$ had practically disappeared in wild-type mice from the region that was previously damaged, whereas their presence persisted in $\Delta I g f 1 r$ mutants (Fig. 4). Thus, counting of $\mathrm{F} 4 / 80^{+}$cells at 9 weeks indicated that their number was at least fourfold higher in the mutants than in the controls (Fig. 4).

Mature oligodendrocyte regeneration is decreased in the absence of IGF signaling

During cuprizone-induced demyelination, the mature oligodendrocyte popula-
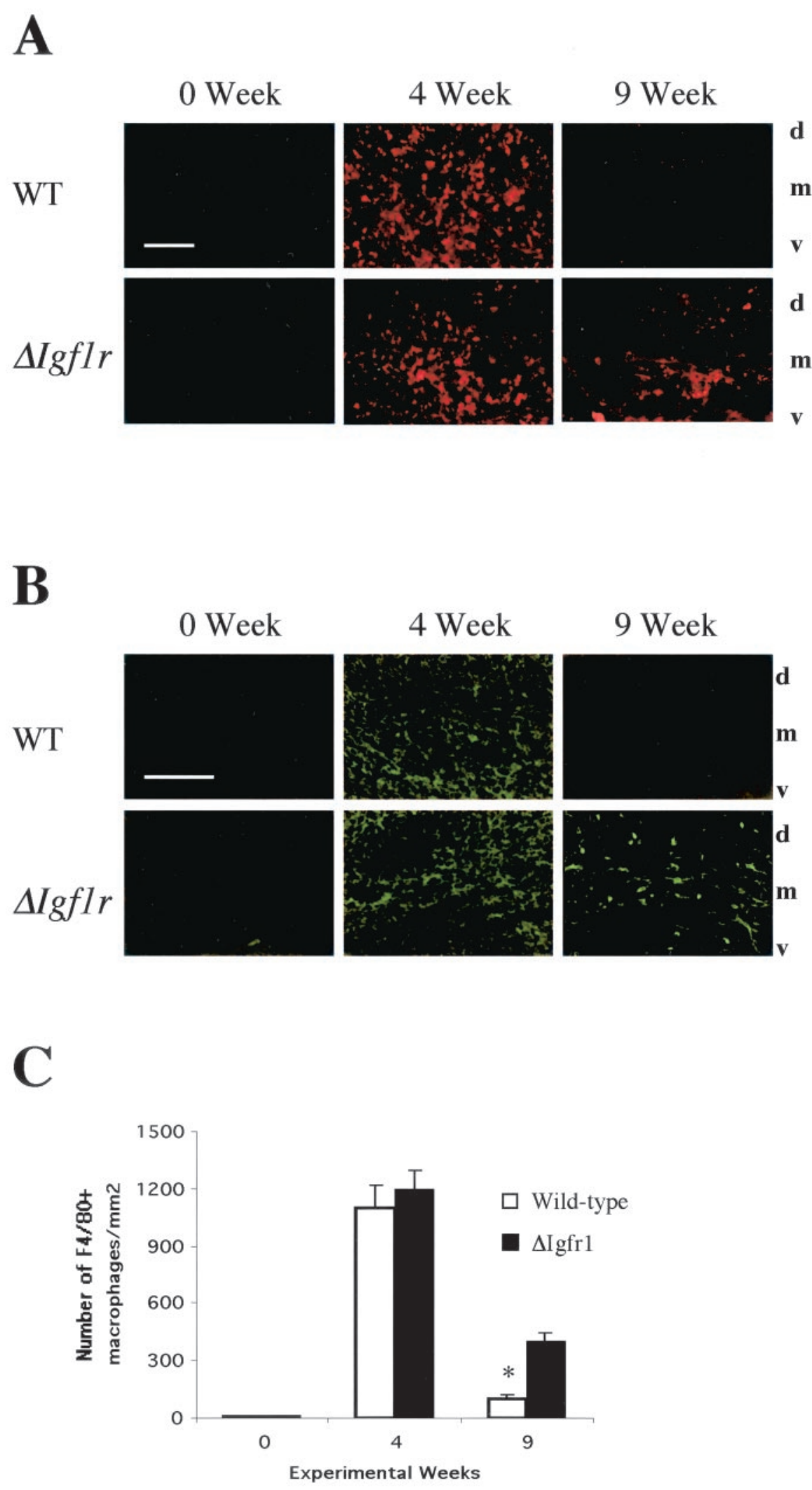

Figure 4. Effects of IGF signaling on the accumulation of microglia/macrophages within the demyelinating and remyelinating lesion. Large numbers of microglia/macrophages ( $A$; red), detected by the ant-F4/80 antibody, and TNF- $\alpha$ ( $B$; green) accumulate within the demyelinating corpus callosum in both wild-type (WT) and $\Delta /$ gfir mice, peaking at 4 weeks. However, although the microglia/macrophages and TNF- $\alpha$ disappear from the remyelinating lesion in WT mice, a large number of microglia/macrophages and TNF- $\alpha^{+}$cells remain within the demyelinated region of the corpus callosum in $\Delta / g f 1 r$ mice at 9 weeks. d, Dorsal; $m$, middle; v, ventral. Scale bars, 50 $\mu \mathrm{m}$. C, The mean and SEM bars for the number of $\mathrm{F} 4 / 80^{+}$macrophages $/ \mathrm{mm}^{2}$ are plotted. ${ }^{*} p<0.01$. 

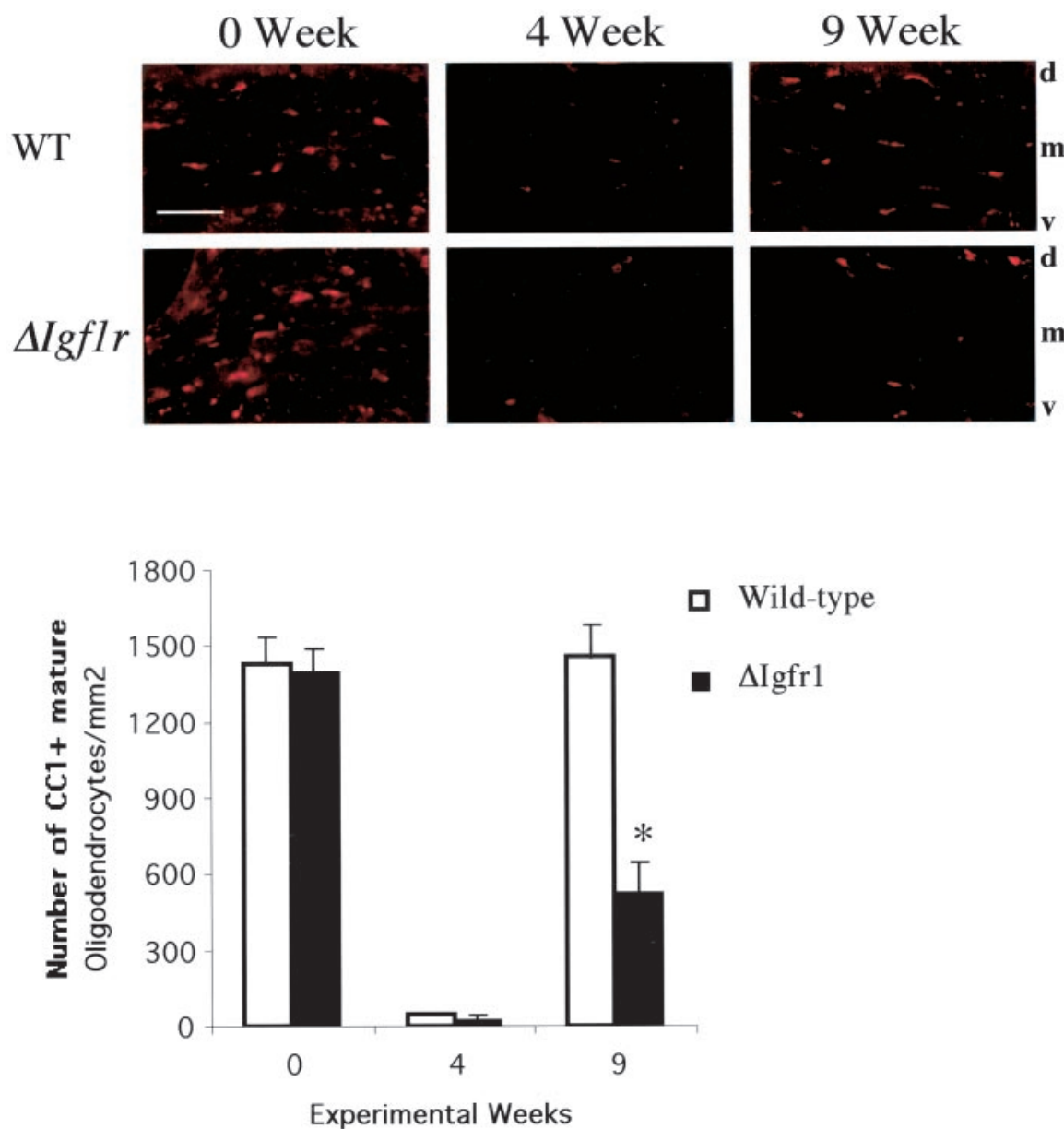

Figure 5. Effects of IGF signaling on the regeneration of the mature oligodendrocyte population after an acute demyelinating lesion. Mature oligodendrocytes (red), detected by the anti-CC 1 antibody, become depleted in both wild-type (WT) and $\Delta / g f 1 r$ mice by 4 weeks. However, the regeneration of mature oligodendrocytes during recovery is dramatically reduced in the absence of IGF signaling for the entire depth of the corpus callosum, and there is most likely even a greater reduction in the middle region. $\mathrm{d}$, Dorsal; $\mathrm{m}$, middle; $v$, ventral. Scale bar, $40 \mu \mathrm{m}$. The mean and SEM bars for the number of $\mathrm{CC}^{+}{ }^{+}$mature oligodendrocytes $/ \mathrm{mm}^{2}$ are plotted. ${ }^{*} p<0.01$.

tion, identified by the $\mathrm{CC} 1$ antibody, disappeared throughout the entire depth of the corpus callosum in both wild-type and $\Delta I g f 1 r$ mice at 4 weeks (Fig. 5) (Mason et al., 2000a). Subsequently, mature oligodendrocytes reappeared in normal numbers in wildtype mice after they were returned to a normal diet after 6 weeks of cuprizone treatment (Fig. 5) (Mason et al., 2000a). In contrast, mature oligodendrocytes did not regenerate to normal numbers in $\Delta \operatorname{Igflr}$ mice at 9 weeks (Fig. 5). Counting of $\mathrm{CC}^{+}{ }^{+}$oligodendrocytes at this time indicated the number of mature oligodendrocytes in the mutants was $\sim 45 \%$ of that found in controls. These cell counts reflect $\mathrm{CC}^{+}{ }^{+}$oligodendrocytes throughout the entire depth of the corpus callosum (counting cells within specific regions of the callosum is very difficult), and after examining many sections, we can state that most of these oligodendrocytes resided in the remyelinated ventral and dorsal regions of the callosum and not in the demyelinated middle region. Additional analysis (TUNEL assay combined with CC1 immunostaining) indicated that the paucity of oligodendrocytes in the middle region was not because of the apoptotic death of newly generated oligodendrocytes during recovery at 6 and 9 weeks (data not shown).
Oligodendrocyte progenitors do not accumulate in the absence of IGF signaling

In wild-type mice, $\mathrm{NG}_{2}{ }^{+}$oligodendrocyte progenitors accumulate within the demyelinated corpus callosum between 3 and 5 weeks of cuprizone feeding and then decline in number during the reappearance of mature oligodendrocytes and remyelination (Fig. 6A,B) (Mason et al., 2000a). In contrast, few $\mathrm{NG}_{2}{ }^{+}$oligodendrocyte progenitors were observed within the middle region of the callosum in $\Delta I g f 1 r$ mice at 4 weeks (Fig. 6A,B). However, there was some accumulation of these progenitors within the dorsal and ventral edges of the callosum, in which regeneration of mature oligodendrocytes and remyelination occurred (Fig. 6A). Thus, the absence of mature oligodendrocytes and lack of remyelination in the middle region of the callosum correlates spatially with the near absence of progenitors at 4 weeks. However, by 9 weeks, there appeared to be a low density of progenitors evenly distributed throughout the corpus callosum in both wild-type and $\Delta I g f 1 r$ mice (Fig. $6 A$ ), even though there was still a significant reduction in the number of oligodendrocyte progenitors throughout the entire corpus callosum in $\Delta \operatorname{Igflr}$ mice (Fig. $6 \mathrm{~B}$ ). Under our conditions, NG2 staining did not colocalize with $\mathrm{F} 4 / 80^{+}$microglia/macrophages as reported previously (Nishiyama et al., 1997).

The lower number of $\mathrm{NG}_{2}{ }^{+}$progenitors within the middle region of the callosum in $\Delta I g f 1 r$ mice could be because of increased cell death and/or hypoproliferation. To address these issues, we used immunohistochemistry to determine whether proliferating (using the KI-67 antibody) and/or apoptotic (TUNEL assay) $\mathrm{NG} 2^{+}$progenitors were present in wild-type and $\Delta I g f 1 r$ mice at 4 weeks. In contrast to the number of proliferating $\mathrm{NG} 2^{+}$progenitors throughout the corpus callosum in wild-type mice $\left(88 \pm 21\right.$ cells $/ \mathrm{mm}^{2}$ ) (Arnett et al., 2001), there was a significant $(p<0.05)$ reduction in the number of proliferating $\mathrm{NG}_{2}{ }^{+}$progenitors throughout the corpus callosum in $\Delta I g f 1 \mathrm{r}$ mice $\left(22 \pm 9\right.$ cells $/ \mathrm{mm}^{2}$ ). All of the proliferating $\mathrm{NG}_{2}{ }^{+}$progenitors were observed within the ventral and dorsal regions of the corpus callosum and not within the middle region in the $\Delta \operatorname{Igflr}$ mice (Fig. $6 C$ ). Apoptotic $\mathrm{NG}^{+}$ progenitors were only observed throughout the corpus callosum in $\Delta \operatorname{Igflr}$ mice $\left(55 \pm 17\right.$ cells $\left./ \mathrm{mm}^{2}\right)$ and not within wild-type mice $\left(0\right.$ cells $/ \mathrm{mm}^{2}$; Fig. $\left.6 D\right)$ (Arnett et al., 2001). It seems, therefore, that the lower number of progenitors within the demyelinating lesion is because of the inability of these cells to survive and then possibly proliferate.

\section{Discussion}

Our observations in the $\Delta I g f 1 \mathrm{r}$ mice demonstrate the importance of IGF signaling through IGF1R in remyelination. This signaling is not required for myelination during development, as shown in 
IGF-1-/- mice (Cheng et al., 1998). In addition, we noted that the lack of IGFR1 in oligodendrocyte progenitor cells does not appear to impede myelination during CNS development. Although we have not examined the kinetics of myelination or counted numbers of progenitors and mature oligodendrocytes in detail, the adult mice appeared neurologically normal, both behaviorally and morphologically. We suggest that other factors may compensate for the absence of IGF signaling through IGFR1 to allow oligodendrocytes to survive and myelinate appropriately. However, remyelination was impaired in these $\Delta$ Igflr mice.

The absence of remyelination in the middle region of the corpus callosum in the $\Delta I g f 1 r$ mice could be because of the loss of IGF signaling in neurons, oligodendrocytes, or both, because the CaMKII $\alpha$ promoter expresses cre in both neurons and oligodendrocyte progenitors in these transgenic mice. Because normalappearing axons (without swelling) were observed within the demyelinated middle region, we presume that the absence of remyelination was not because of axonal degeneration. Although we do not know whether the $\Delta \operatorname{Igflr}$ mice retain the full complement of axons after cuprizone treatment, the degree of NF-H immunoreactivity appears similar to that in wild-type mice. In addition, it would appear that the absence of IGF signaling in neurons does not affect the ability of their axons to become remyelinated, because remyelination was observed in the dorsal and ventral regions. In contrast, the absence of oligodendrocyte progenitors and mature oligodendrocytes within the middle region of the callosum correlates spatially with the absence of remyelination in the $\Delta I g f 1 r$ mice. Thus, we postulate that IGF signaling within oligodendrocytes is needed for remyelination.

The belief that IGF-1 promotes spontaneous remyelination of the adult CNS following a demyelinating insult has been widely speculated. Systemic administration of IGF-1 enhances remyelination by inhibiting the immune response in some autoimmune models of demyelination (Liu et al., 1997; Li et al., 1998), but not in others (Cannella et al., 2000). Although IGF-1 induces the differentiation of oligodendrocyte progenitors in vitro (McMorris and Dubois-Dalq, 1988; Mozell and McMorris, 1991; Barres et al., 1993) and is upregulated within remyelinating lesions in cuprizone-, experimental autoimmune encephalomyelitis-, and lysolecithininduced demyelination (Liu et al., 1994;
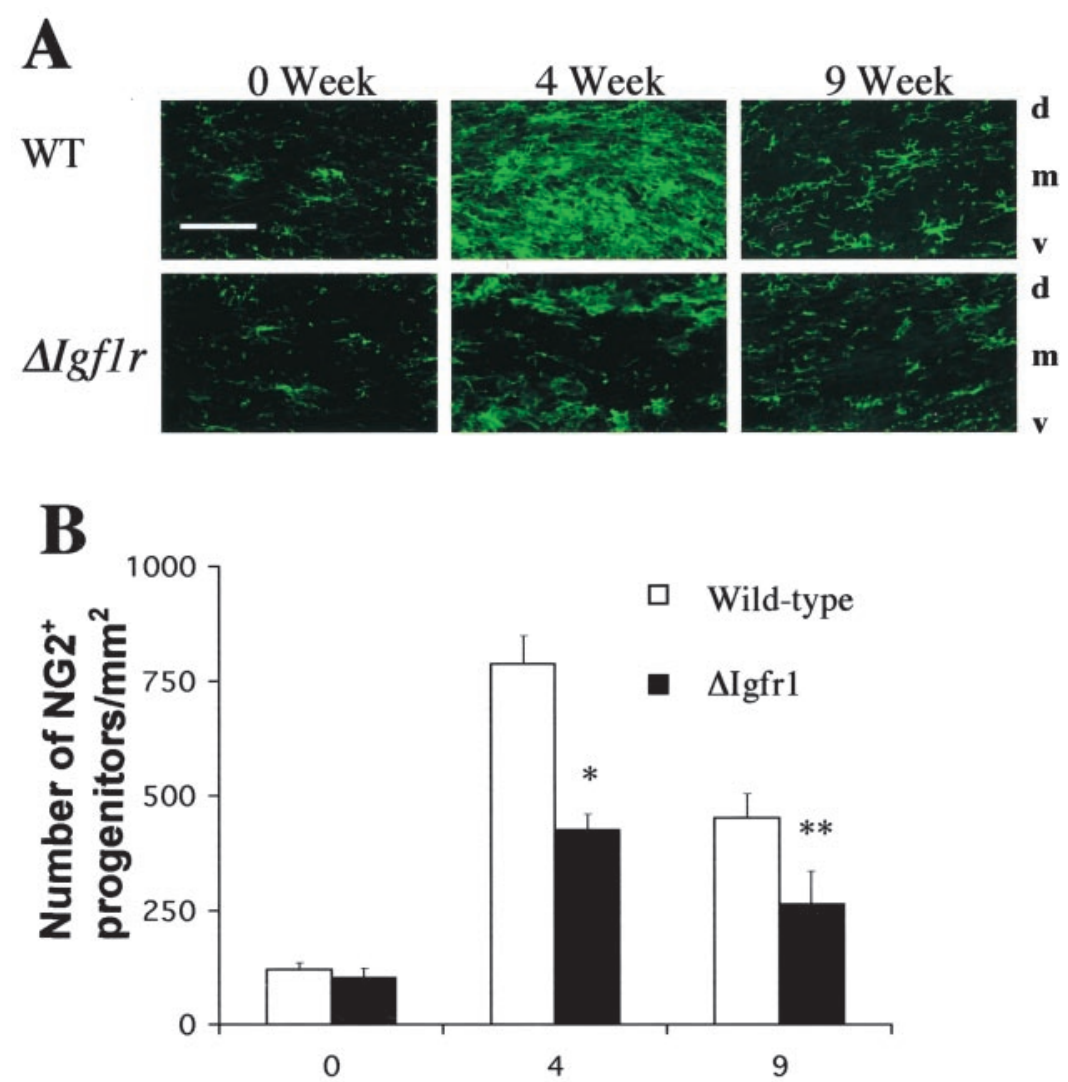

Experimental Weeks

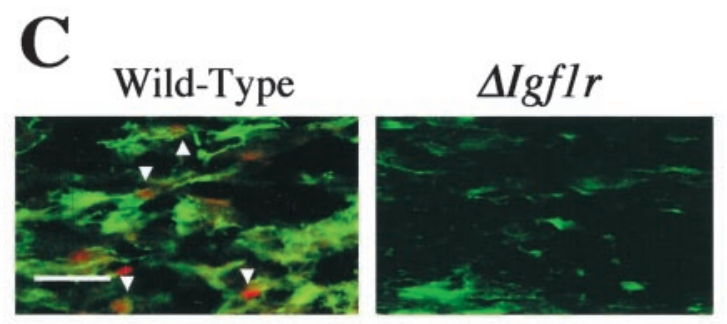

D
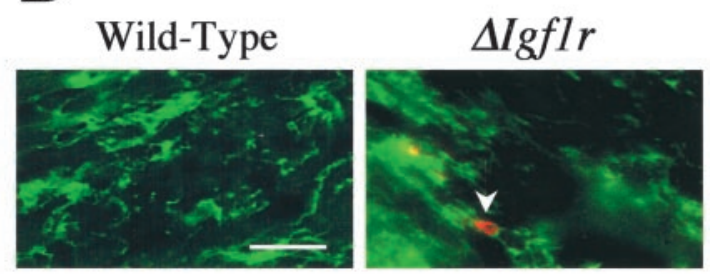

Figure 6. Effects of IGF signaling on the accumulation of oligodendrocyte progenitors. A, Oligodendrocyte progenitors (green), detected by NG2, accumulate with the demyelinating corpus callosum in wild-type (WT) mice, peaking at 4 weeks when demyelination is complete. However, oligodendrocyte progenitors only accumulate within the ventral (v) and dorsal (d) edges of the corpus callosum, with very few cells in the middle $(\mathrm{m})$ region of the corpus callosum in $\Delta / g f 1 \mathrm{r}$ mice. By 9 weeks, there is a dramatic reduction in the number of $\mathrm{NG}^{+}$oligodendrocyte progenitors within the corpus callosum of WT and $\Delta /$ gfir mice. $B$, The mean and SEM bars for the number of $\mathrm{NG}^{+}{ }^{+}$oligodendrocyte progenitors $/ \mathrm{mm}^{2}$ throughout the entire depth of the corpus callosum are plotted. ${ }^{*} p<0.01$; ${ }^{* *} p<0.05$. C, Although a large number of NG2 ${ }^{+}$oligodendrocyte progenitors (green) proliferate, detected by an anti-KI-67 antibody (red), at 4 weeks within the demyelinated corpus callosum in wild-type mice (arrowheads indicate NG2 ${ }^{+} / \mathrm{KI}_{-} 67^{+}$cells), no proliferating NG2 ${ }^{+}$oligodendrocyte progenitors were observed in the demyelinated corpus callosum of $\Delta / g f 1 r$ mice at 4 weeks. Three nonadjacent sections from each of three different wild-type and $\Delta / g f 1 r$ mice were examined for proliferating NG2 ${ }^{+}$oligodendrocyte progenitors. D, Apoptotic [detected by TUNEL assay (red)] NG2 ${ }^{+}$oligodendrocyte progenitors (green) were observed within the middle region of the corpus callosum in $\Delta / g f 1 r$ mice at 4 weeks (arrowhead indicates apoptotic NG2 ${ }^{+}$cells), whereas no apoptotic progenitors were detected in wild-type mice. Three nonadjacent sections from each of three different wild-type and CaMKIl $\alpha$-cre/lgf1r $\Delta^{\text {flox/- }}$ mice were examined for apoptotic $\mathrm{NG} 2^{+}$oligodendrocyte progenitors. Scale bars: $A, 50 \mu \mathrm{m} ; C, 15 \mu \mathrm{m} ; D, 10 \mu \mathrm{m}$. 
Hinks and Franklin, 1999; Mason et al., 2000a), there has been no evidence to support the theory that IGF-1 promotes remyelination by inducing the differentiation of oligodendrocyte progenitors (O'Leary et al., 2002). In contrast, increased levels of IGF-1 protect mature oligodendrocytes within demyelinating lesions, thus facilitating a rapid recovery (Mason et al., 2000b). In addition, we show here that IGF signaling is required for the survival, proliferation, and differentiation of oligodendrocyte progenitors during remyelination.

We suggest that the increased death of oligodendrocyte progenitors in the $\Delta \operatorname{Igf} 1 \mathrm{r}$ mice is related to their inability to respond to IGF-1 as a survival factor (Barres et al., 1993; Ness and Wood, 2002). Cytotoxic factors, such as TNF- $\alpha$, that normally increase and then decrease with the cycle of demyelination and remyelination (Arnett et al., 2001) remain elevated within the middle region of the demyelinating lesion in $\Delta \operatorname{Igf} 1 \mathrm{r}$ mice, most likely because of the continued presence of macrophages and some astrocytes (Arnett et al., 2001). TNF- $\alpha$ appears to have two opposite effects on oligodendrocytes and their progenitors, each mediated by a distinct TNF receptor (TNFR), both of which are upregulated during inflammatory conditions (Tchelingerian et al., 1995; Dopp et al., 1997). TNF- $\alpha$ produces cell death through TNFR1 (Haridas et al., 1998; Weiss et al., 1998; Ashkenazi and Dixit, 1999), but it also induces the proliferation of oligodendrocyte progenitors through TNFR2 (Arnett et al., 2001). It is possible that in the absence of IGF signaling, the balance of effects induced by TNF- $\alpha$ is tilted toward cell death, and, thus, oligodendrocytes succumb to the toxic effects of TNF- $\alpha$ rather than surviving and manifesting the proliferative effects. In fact, IGF-1 inhibits the death of oligodendrocyte progenitors induced by TNF- $\alpha$ in vitro (Ye and D'Ercole, 1999). Interestingly, remyelination occurred in the dorsal and ventral zones of the callosum, in which macrophages and their associated cytokines disappeared according to the normal time schedule (Arnett et al., 2001; Matsushima and Morell, 2001). It is, therefore, possible that there is a reduced level of TNF- $\alpha$ in the dorsal and ventral zones compared with the middle, and, thus, progenitor survival and remyelination are better preserved at the edges of the callosum, even in the absence of IGF signaling.

Although IGF1R expression is not disrupted within microglia and macrophages (data not shown), these cells remain within the lesion in $\Delta I g f 1 r$ mice, even after the mice are returned to a normal diet. The stimulus that keeps the macrophages within the lesion and the effect their presence has on the inability of the lesion to remyelinate is not known. Because we did not find evidence for recurrent remyelination and demyelination, we do not believe that the macrophages remained to clear debris from failed attempts at remyelination. It is possible that macrophages remain in the area because of continued progenitor cell death. We did observe small numbers of $\mathrm{NG}_{2}{ }^{+}$cells in the middle region of the callosum after 9 weeks (Fig. 6A), and although we did not detect TUNEL $^{+}$cells in that population, we cannot rule out the possibility of a slow continuation of progenitor death.

Although spontaneous remyelination occurs after a demyelinating insult to the adult CNS, it is often incomplete, presumably because of the depletion of oligodendrocyte progenitors (Lucchinetti et al., 1996; Blakemore and Keirstead, 1999), the loss of axons, the absence of an environment to support progenitor differentiation (Wolswijk, 1998; Chang et al., 2002), or a combination of the above events. The present work, when combined with previous results demonstrating the ability of IGF-1 to prevent mature oligodendrocyte death and depletion during acute demyelination (Mason et al., 2000b), suggests that IGF signaling is vital for not only facilitating recovery from acute demyelinating insults, but that therapies incorporating increased levels of IGF-1 may prevent the death and depletion of oligodendrocytes and formation of chronic lesions. Further investigation of IGF signaling in preventing the formation of chronic demyelinating lesions is warranted.

\section{References}

Arnett HA, Mason JL, Marino M, Suzuki K, Matsushima GK, Ting JP-Y (2001) TNF $\alpha$ promotes proliferation of oligodendrocyte progenitors and remyelination. Nat Neurosci 4:1116-1122.

Ashkenazi A, Dixit VM (1999) Apoptosis control by death and decoy receptors. Curr Opin Cell Biol 11:255-260.

Barres BA, Schmid R, Sendnter M, Raff MC (1993) Multiple extracellular signals are required for long-term oligodendrocyte survival. Development 118:283-295.

Blakemore WF, Keirstead HS (1999) The origin of remyelinating cells in the central nervous system. J Neuroimmunol 98:69-76.

Cannella B, Pitt D, Capello E, Raine CS (2000) Insulin-like growth factor-1 fails to enhance central nervous system myelin repair during autoimmune demyelination. Am J Pathol 157:933-943.

Chang A, Tourtellotte WW, Rudick R, Trapp BD (2002) Premyelinating oligodendrocytes in chronic lesions of multiple sclerosis. N Engl J Med 346:165-173.

Cheng CM, Joncas G, Reinhardt RR, Farrer R, Quarles R, Janssen J, McDonald MP, Crawley JN, Powell-Braxton L, Bondy CA (1998) Biochemical and morphometric analyses show that myelination in the insulin-like growth factor 1 null brain is proportionate to its neuronal composition. J Neurosci 18:5673-5681.

Di Cola G, Cool MH, Accili D (1997) Hypoglycemic effect of insulin-like growth factor-1 in mice lacking insulin receptors. J Clin Invest 99:2538-2544.

Dietrich P, Dragatsis I, Xuan S, Zeitlin S, Efstratiadis A (2000) A conditional mutagenesis in mice with heat shock promoter-driven cre transgenes. Mamm Genome 11:196-205.

Dopp JM, Mackenzie-Graham A, Otero GC, Merrill JE (1997) Differential expression, cytokine modulation, and specific functions of type- 1 and type-2 tumor necrosis factor receptors in rat glia. J Neuroimmunol 75:104-112.

Dragatsis I, Zeitlin S (2000) CamKII $\alpha$-cre transgene expression and recombination patterns in the mouse brain. Genesis 26:133-135.

Gensert JM, Goldman JE (1997) Endogenous progenitors remyelinate demyelinated axons in the adult CNS. Neuron 19:197-203.

Gensert JM, Goldman JE (2001) Heterogeneity of cycling progenitors in the adult mammalian cortex and white matter. J Neurobiol 48:75-86.

Haridas V, Darnay BG, Natarajan K, Heller R, Aggarwal BB (1998) Overexpression the p80 TNF receptor leads to TNF-dependent apoptosis, nuclear factor $-\kappa \mathrm{B}$ activation, and $\mathrm{c}$-Jun kinase activation. J Immunol 160:3152-3162.

Hinks GL, Franklin RJ (1999) Distinctive patterns of PDGF-A, FGF-2, IGF-I, and TGF- $\beta 1$ gene expression during remyelination of experimentally-induced spinal cord demyelination. Mol Cell Neurosci 14:153-168.

Hiremath MM, Saito Y, Knapp GW, Ting JP-Y, Suzuki K, Matsushima GK (1998) Microglial/macrophage accumulation during cuprizone-induced demyelination in C57BL/6 mice. J Neuroimmunol 92:38-49.

Li W, Quigley L, Yao D-L, Hudson LD, Brenner M, Zhang BJ, Brocke S, McFarland HF, Webster HD (1998) Chronic relapsing experimental autoimmune encephalomyelitis: effects of insulin-like growth factor-I treatment on clinical deficits, lesion severity, glial responses, and blood brain barrier defects. J Neuropathol Exp Neurol 57:426-438.

Liu J-P, Baker J, Perkins AS, Robertson EJ, Efstratiadis A (1993) Mice carrying null mutations of the genes encoding the insulin-like growth factor 1 (igf1) and type-1 IGF receptor (igf1r). Cell 75:59-72.

Liu X, Yao D-L, Bondy CA, Brenner M, Hudson LD, Zhou J, Webster Hd (1994) Astrocytes express insulin-like growth factor-1 (IGF-1) and its binding protein, IGFBP-2, during demyelination induced by experimental autoimmune encephalomyelitis. Mol Cell Neurosci 5:418-430.

Liu X, Linnington C, Webster HD, Lassmann S, Yao DL, Hudson LD, Wekerle H, Kreutzberg GW (1997) Insulin-like growth factor-I treatment reduces immune cell responses in acute non-demyelinative experimental autoimmune encephalomyelitis. J Neurosci 47:531-538. 
Lucchinetti CF, Bruck W, Rodriguez M, Lassmann H (1996) Distinct patterns of multiple sclerosis pathology indicates heterogeneity in pathogenesis. Brain Pathol 6:259-274.

Ludwin SK (1978) Central nervous system demyelination and remyelination in the mouse. Lab Invest 39:597-612.

Mason JL, Goldman JE (2002) $\mathrm{A}^{2} \mathrm{~B}^{+}{ }^{+}$and $\mathrm{O} 4{ }^{+}$cycling progenitors in the adult forebrain white matter respond differentially to PDGF-AA, FGF-2 and IGF-1. Mol Cell Neurosci 20:30-42.

Mason JL, Jones JJ, Taniike M, Suzuki K, Morell P, Matsushima GK (2000a) Mature oligodendrocyte apoptosis precedes IGF-1 production and oligodendrocyte progenitor accumulation and differentiation during demyelination/remyelination. J Neurosci Res 61:251-262.

Mason JL, Ye P, Suzuki K, D’Ercole AJ, Matsushima GK (2000b) Insulinlike growth factor-1 inhibits mature oligodendrocyte apoptosis during primary demyelination. J Neurosci 20:5703-5708.

Mason JL, Suzuki K, Chaplin DD, Matsushima GK (2001a) Interleukin1beta promotes repair of the CNS. J Neurosci 21:7046-7052.

Mason JL, Langaman C, Morell P, Suzuki K, Matsushima GK (2001b) Episodic demyelination and subsequent remyelination within the murine central nervous system: changes in axonal calibre. Neuropathol Appl Neurobiol 27:50-58.

Matsushima GK, Morell P (2001) The neurotoxicant, cuprizone, as a model to study demyelination and remyelination in the central nervous system. Brain Pathol 11:107-116.

McMorris FA, Dubois-Dalq M (1988) Insulin-like growth factor 1 promotes cell proliferation and oligodendroglial commitment in rat glial progenitor cells developing in vitro. J Neurosci Res 21:199-209.

Messersmith DJ, Murtie JC, Le TQ, Frost EE, Armstrong RC (2000) Fibroblast growth factor 2 (FGF2) and FGF receptor expression in an experimental demyelinating disease with extensive remyelination. J Neurosci Res 62:241-256.

Mozell RL, McMorris FA (1991) Insulin-like growth factor I stimulates oligodendrocyte development and myelination in rat aggregate cultures. J Neurosci Res 30:382-390.

Ness JK, Wood TL (2002) Insulin-like growth factor I, but not neurotrophin-3, sustains Akt activation and provides long-term protection of immature oligodendrocytes from glutamate-mediated apoptosis. Mol Cell Neurosci 20:476-488.

Nishiyama A, Lin X-H, Giese N, Heldin C-H, Stallcup WB (1996) Colocalization of NG2 proteoglycan and PDGF $\alpha$-receptor on O2A progenitor cells in the developing rat brain. J Neurosci Res 43:299-314.
Nishiyama A, Yu M, Drazaba JA, Tuohy VK (1997) Normal and reactive $\mathrm{NG} 2{ }^{+}$glial cells are distinct from resting and activated microglia. J Neurosci Res 48:299-312.

O’Leary MT, Hinks GL, Charlton HM, Franklin RJM (2002) Increasing local levels of IGF-1 mRNA expression using adenoviral vectors does not alter oligodendrocyte remyelination in the CNS of aged rats. Mol Cell Neurosci 19:32-42.

Redwine JM, Armstrong RC (1998) In vivo proliferation of oligodendrocyte progenitors expressing PDGF $\alpha$ R during early remyelination. J Neurobiol 37:413-428.

Rossant J, McMahon A (1999) "Cre"-ating mouse mutants-a meeting review on conditional mouse genetics. Genes Dev 13:142-145.

Sauer B (1998) Inducible gene targeting in mice using the Cre/lox system. Methods 14:381-392.

Sidman RL, Abervine JB, Pierce ET (1971) Atlas of the mouse brain and spinal cord. Cambridge, MA: Harvard UP.

Soriano P (1999) Generalized lacZ expression with the ROSA26 Cre reporter strain. Nat Genet 21:70-71.

Tchelingerian JL, Monge M, Le Saux F, Zalc B, Jacque C (1995) Differential oligodendroglial expression of the tumor necrosis factor receptors in vivo and in vitro. J Neurochem 65:2377-2380.

Vallano ML, Beaman-Hall CM, Mathur A, Chen Q (2000) Astrocytes express specific variants of CaM KII delta and gamma, but not alpha and beta, that determine their cellular localizations. Glia 30:154-164.

Weiss T Grell M, Siemienski K, Muhlenbeck F, Durkop H, Pfizenmaier K, Scheurich P, Wajant H (1998) TNFR80-dependent enhancement of TNFR60-induced cell death is mediated by TNFR-associated factor 2 and is specific for TNFR60. J Immunol 161:3136-3142.

Wolswijk G (1998) Chronic stage multiple sclerosis lesions contain a relatively quiescent population of oligodendrocyte precursor cells. J Neurosci 18:601-609.

Yao D-L, West NR, Bondy CA Brenner M, Hudson LD, Zhou J, Collins GH, Webster HD (1995) Cryogenic spinal cord injury induces astrocytic gene expression of insulin-like growth factor 1 and insulin-like growth factor binding protein 2 during myelin regeneration. J Neurosci Res 40:647-659.

Ye P, D’Ercole AJ (1999) Insulin-like growth factor I protects oligodendrocytes from tumor necrosis factor- $\alpha$-induced injury. Endocrinology 140: 3063-3072. 